\title{
TMV Infection Cycle in Plant Cells Revealed by 3-D Microscopy
}

\author{
R.H. Berg, S. Asurmendi, R.N. Beachy
}

Donald Danforth Plant Science Center, 975 North Warson Road, St. Louis, MO 63132

Three-dimensional microscopy has been essential in our investigation of infection of plant cells by tobacco mosaic virus (TMV). The genome of TMV encodes a protein essential for replication of the genome (replicase), a $30 \mathrm{kDa}$ movement protein (MP) that is essential for cell-cell spread of infection, and a $17.5 \mathrm{kDa}$ capsid protein (CP). The use of green fluorescent protein (GFP) from Aequoria victoria fused with MP or CP made it possible to collect images of the progression of infection and led to a more complete understanding of how cells react to infection and alter their biochemical activities. Confocal microscopy has been indispensable in this work, providing high contrast 3-D imaging of the cellular distribution of virus proteins [1-6]. For the most part, studies have been conducted with protoplasts isolated from cultured tobacco BY-2 cells, with complementary studies in intact leaf tissue.

Presently we are colocalizing all three viral proteins in the same cell using immunofluorescence and 3-D microscopy (confocal optical sections and 3-D digital reconstructions). Sampling different times after infection, we have analyzed the spatialtemporal distribution of viral replicase in relation to movement protein and coat protein (Fig. 1).

We are using spectral imaging (with the Zeiss Meta detector on the LSM 510) to colocalize the three virus proteins with respect to various plant cell components. Preliminary results with the Meta detector show it can separate chlorophyll emission from that of DS Red (Fig. 2), with which it overlaps considerably. It is difficult to discriminate these signals using the conventional approach with emission filters. Using this detector we have also been able to image CFP, GFP and YFP in the same cells (Fig. 3). Experiments are now planned to colocalize the three viral proteins (immunofluorescent) with several fluorescent proteins (organelle-targeted GFP vriants) and the plant cytoskeleton (immunofluorescent microtubules and actin stained with phalloidin-rhodamine).

[1] M. Heinlein et al., Plant Cell 10 (1998) 1107

[2] P. Mas and R.N. Beachy, Plant J. 15 (1998) 835

[3] P. Mas and R.N. Beachy, J. Cell Biol. 147 (1999) 945

[4] P. Mas and R.N. Beachy, Proc. Natl. Acad. Sci. 97 (2000) 12345

[5] S.G. Lazarowitz and R.N. Beachy, Plant Cell 11 (1999) 535

[6] M. Bendahmane et al., Proc. Natl. Acad. Sci. (2002), in press

[7] Supported by grants to RNB from NIH (No. 1R01GM/A162979-01 and No. 2R01AI27161-09A2) 


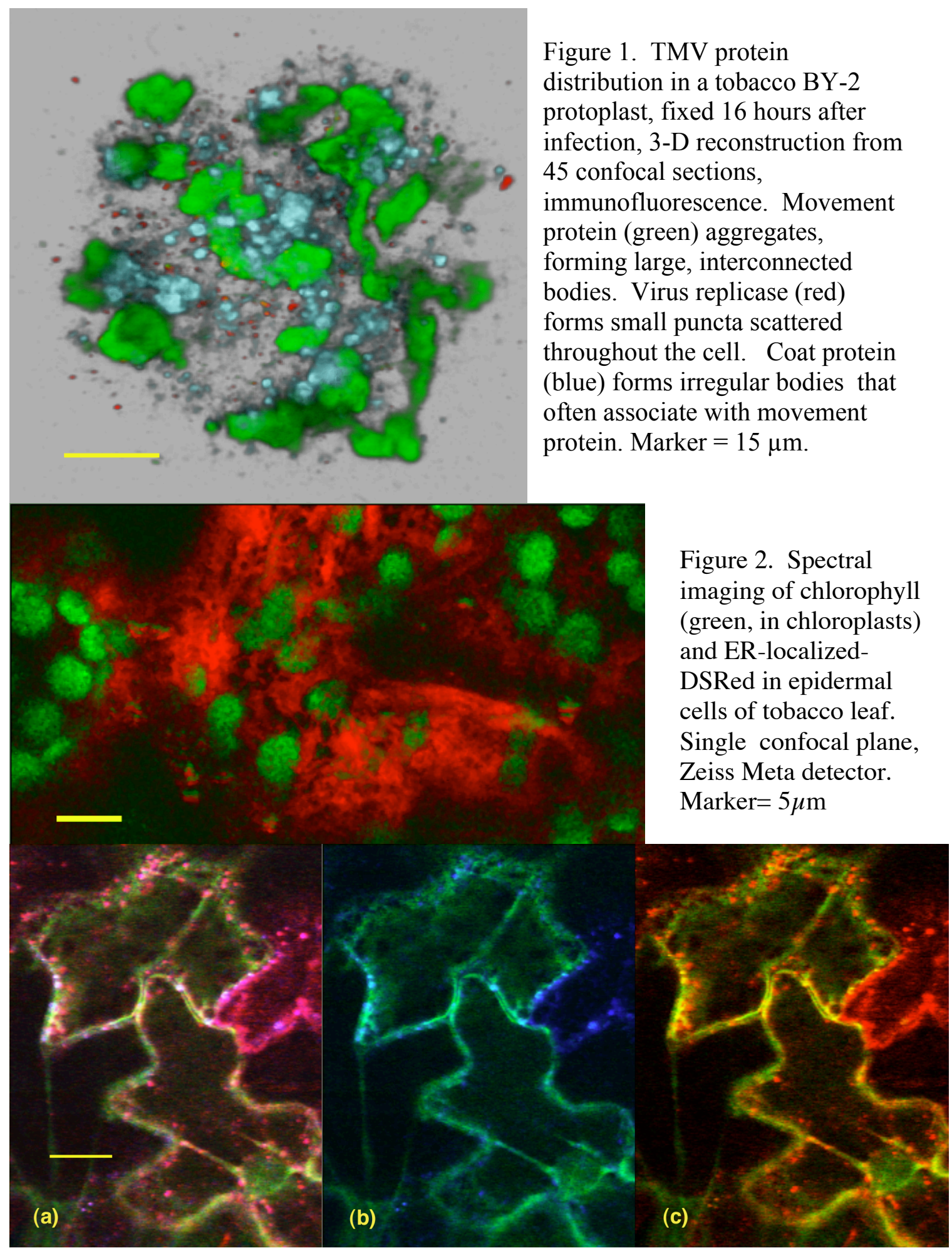

Figure 3. Spectral imaging of Rab5-localized YFP (red), ER-localized CFP (green) and TMV MP:GFP (blue) in tobacco leaf epidermal cells. Transient expression via Agrobacterium tumefaciens, all three fluorescent proteins were expressed in two of these cells. (a) three colors, (b) blue and green, (c) red and green. Spectral imaging in this single confocal plane was done using the Zeiss Meta detector. Marker $=15 \mu \mathrm{m}$. 